\title{
Cupins de duas florestas de restinga do nordeste brasileiro
}

\author{
Alexandre Vasconcellos ${ }^{1}$, Ana Cerilza S. Mélo ${ }^{2,3}$, Eusébio de M. Vasconcelos Segundo ${ }^{1}$ \\ \& Adelmar G. Bandeira ${ }^{1}$
}

1. Departamento de Sistemática e Ecologia, CCEN, Universidade Federal da Paraíba, 58051-900 João Pessoa, Paraíba, Brasil.
(alexvasconcellos@ @ahoo.com.br)
2. Programa de Pós-Graduação em Ciências Biológicas (Zoologia), UFPB, João Pessoa, PB, Brasil.
3. Departamento de Biologia, Universidade Estadual de Feira de Santana, 44031-460 Feira de Santana, Bahia, Brasil.

ABSTRACT. Termites from two restinga forests of Northeastern Brazil. The structure of termite communities was evaluated at two restinga forests (a characteristic type of vegetation occurring on nutrient-poor sandy soils along the Brazilian coastline), located in the municipalities of Mataraca and Cabedelo, State of Paraíba. A standardised sampling protocol was used in both sites. Twenty-five species were found, 19 of them at Mataraca and 15 at Cabedelo, with just 9 species in common to both sites. Species of Nasutitermitinae and wood-feeding groups were dominant at both study sites. The low species richness and frequency of humus-feeders species, and species of the subfamily Apicotermitinae as well, seem to be related to the restinga soil properties. The conspicuous nest (all arboreal) builders were Armitermes holmgreni Snyder, 1926, Microcerotermes exiguus (Hagen, 1858), M. strunckii (Sörensen, 1884), Nasutitermes corniger (Motschulsky, 1855), N. ephratae (Holmgren, 1910), and N. macrocephalus (Silvestri, 1903). The termite fauna of the studied restinga was composed by typical species of other Brazilian ecosystems, such as Atlantic forest and Cerrado, being in agreement with the general pattern of distribution set up by the plant communities and the fauna of vertebrates described elsewhere for other Brazilian restingas.

KEYWORDS. Isoptera, biodiversity, geographical distribution, feeding groups, nests.

RESUMO. A estrutura da comunidade de cupins foi avaliada em duas florestas de restinga localizadas nos municípios de Mataraca e Cabedelo, Estado da Paraíba. Um protocolo padronizado de amostragem foi aplicado em cada área. Vinte e cinco espécies foram encontradas, sendo 19 em Mataraca e 15 em Cabedelo, com 9 espécies comuns às duas localidades. As espécies de Nasutitermitinae e as do grupo dos comedores de madeira foram dominantes em ambas as áreas. A baixa riqueza de espécies, em comparação com outros ecossistemas do Nordeste, e a baixa frequiência de encontros de humívoros e da subfamília Apicotermitinae podem estar relacionadas com as propriedades do solo das restingas. As espécies construtoras de ninhos conspícuos (todos arborícolas) foram Armitermes holmgreni Snyder, 1926, Microcerotermes exiguus (Hagen, 1858), M. strunckii (Sörensen, 1884), Nasutitermes corniger (Motschulsky, 1855), N. ephratae (Holmgren, 1910), e N. macrocephalus (Silvestri, 1903). A fauna mostrou-se composta por espécies características de outras formações vegetais, principalmente Mata Atlântica e Cerrado, neste caso estando de acordo com o padrão geral de distribuição estabelecido pelas comunidades vegetais e pela fauna de vertebrados estudados em outras restingas brasileiras.

PALAVRAS-CHAVE. Isoptera, biodiversidade, distribuição geográfica, grupos alimentares, ninhos.

Os cupins são organismos importantes para a manutenção da dinâmica dos processos de decomposição da necromassa vegetal e para os fluxos de nutrientes nas florestas tropicais, devido principalmente à variedade de seus hábitos alimentares e abundância de suas populações (MATSUmoto, 1976; Bignell \& EGGLETon, 2000). Além disso, o comportamento construtor dos cupins causa modificações na estrutura dos solos, promovendo um aumento de porosidade e do transporte de partículas minerais para a superfície e vice-versa (LEE \& WoOD, 1971; WoOD \& SANDS, 1978). As alterações na estrutura dos ecossistemas, causadas pela atividade dos cupins, podem influenciar a disponibilidade de recursos para outros organismos de categorias tróficas diferentes. Por isso, esses insetos são considerados como "engenheiros" de ecossistemas (LAVelLe et al., 1997).

O complexo Mata Atlântica é composto por várias formações vegetais, destacando-se a Mata Atlântica propriamente dita e os ecossistemas associados, como as restingas, manguezais e brejos de altitude (matas serranas inseridas no domínio da caatinga). As restingas são formações vegetais existentes sobre depósitos arenosos presentes ao longo da costa brasileira, cuja fisionomia varia desde herbácea raptante praiana até a florestal fechada (ARAUJo \& LACERDA, 1987). A formação desses depósitos está relacionada com os eventos de transgressão e regressão dos oceanos, ocorridos durante o Quaternário (Bigarella, 1965). No nordeste brasileiro, os ecossistemas de restinga estão localizados na sua maioria sobre a formação geológica do Grupo Barreiras (MABESOONE \& CASTRO, 1975).

Análises da estrutura florística e da fauna de vertebrados demonstraram que as restingas representam uma extensão da distribuição de muitas espécies que ocorrem em outros ecossistemas, como Floresta Amazônica, Mata Atlântica, Caatinga e Cerrado. Desta forma, o número de espécies endêmicas é baixo, estando este fato geralmente relacionado com a idade recente de sua formação (CERQUeIRA, 2000; Rocha, 2000). Em relação aos invertebrados, há uma carência de estudos comparativos entre a fauna das restingas com a de outras formações vegetais que possam testar o padrão geral estabelecido pelas comunidades vegetais e de vertebrados. Tendo isto em vista, objetiva-se analisar as comunidades de cupins de duas florestas de restinga do litoral do Estado da Paraíba e comparar as suas estruturas com a de outros ecossistemas brasileiros.

\section{MATERIAL E MÉTODOS}

O estudo foi desenvolvido em duas florestas de restinga do Estado da Paraíba de abril de 2002 a junho de 
2003 (fig. 1). A primeira, conhecida como Mata da AMEM ( $7^{\circ} 3$ 'S; 34 $\left.51^{\circ} \mathrm{W}\right)$, localiza-se no município de Cabedelo, com área de 103,4 ha e altitude máxima de $10 \mathrm{~m}$. A segunda, pertencente à Mineradora Millennium Inorganic Chemicals do Brasil S.A., situa-se no município de Mataraca (6 $6^{\circ} 9^{\prime}$ S; $34^{\circ} 56^{\prime} \mathrm{W}$ ), extremo norte do Estado, e abrange área de aproximadamente 800 ha, com altitude de até $100 \mathrm{~m}$.

A vegetação de Cabedelo possui árvores de baixo a médio porte (4 a $16 \mathrm{~m})$, mas alguns indivíduos emergentes podem alcançar até $21 \mathrm{~m}$ de altura. A área de Mataraca é constituída por árvores relativamente baixas (4 a $10 \mathrm{~m}$ ), podendo eventualmente apresentar indivíduos com altura máxima de 15 m (Oliveira-Filho \& CARVAlho, 1993).

Em ambas as áreas, as condições climáticas e as características do solo são bastante semelhantes. O clima é tropical e chuvoso (Am de Köppen), com a estação seca geralmente não ultrapassando três meses (NIMER, 1979; Governo do Estado dA PARAíBA, 1985). A temperatura média anual fica entre 24 e $27^{\circ} \mathrm{C}$ e a precipitação entre 1700 e 2000 mm (Governo do Estado da Paraíba, 1985; Carvalho, 1982; Oliveira-Filho \& Carvalho, 1993). O solo é predominantemente arenoso, mineralogicamente pobre, com baixa capacidade de retenção de água.

Para o levantamento da riqueza de espécies foi aplicado o mesmo protocolo padronizado de amostragem termítica descrito em SENA et al. (2003). O protocolo consiste na demarcação aleatória de seis transectos de 65 x 2 m, distribuídos pela área de estudo, em locais com ausência aparente de distúrbio antrópico recente. Em cada transecto são estabelecidas cinco parcelas de $5 \times 2 \mathrm{~m}$, com distância de $10 \mathrm{~m}$ entre elas, totalizando 30 parcelas $\left(300 \mathrm{~m}^{2}\right)$ por localidade. $O$ tempo de coleta em cada parcela é de 1h/pessoa. Nesse período, os cupins são procurados no solo (até cerca de $15 \mathrm{~cm}$ de profundidade), em ninhos ativos e abandonados, troncos e galhos caídos, no folhiço, sob cascas de árvores, raízes mortas, etc.

A identificação em nível genérico foi feita mediante a utilização de chave dicotômica proposta por Constantino (1999). Em nível de espécies, foi consultada a bibliografia listada no Catálogo de Constantino (1998), seguida de comparação com exemplares da Coleção Entomológica do Departamento de Sistemática e Ecologia (DSE) da Universidade Federal da Paraíba (UFPB). Todo o material termítico coletado está depositado na Coleção Entomológica do DSE/UFPB.

Com base em observações de consumo in situ e em informações disponíveis na literatura sobre os cupins neotropicais (BANDeIRA, 1989; Constantino, 1992; De Souza \& Brown, 1994; Bandeira \& Vasconcellos, 2002; BANDEIRA et al., 2003; SENA et al., 2003), as espécies foram categorizadas de acordo com o hábito alimentar em: (1) consumidoras de madeira, que consomem madeira de árvore viva ou nos mais variados estágios de decomposição; (2) consumidoras de húmus, que se alimentam de húmus e geralmente vivem no perfil do solo; (3) consumidoras de madeira e húmus, que consomem húmus e madeira em estágio avançado de decomposição; e (4) consumidoras de madeira e folhas, que consomem tanto madeira em estágio avançado de decomposição como também folhas da serapilheira.

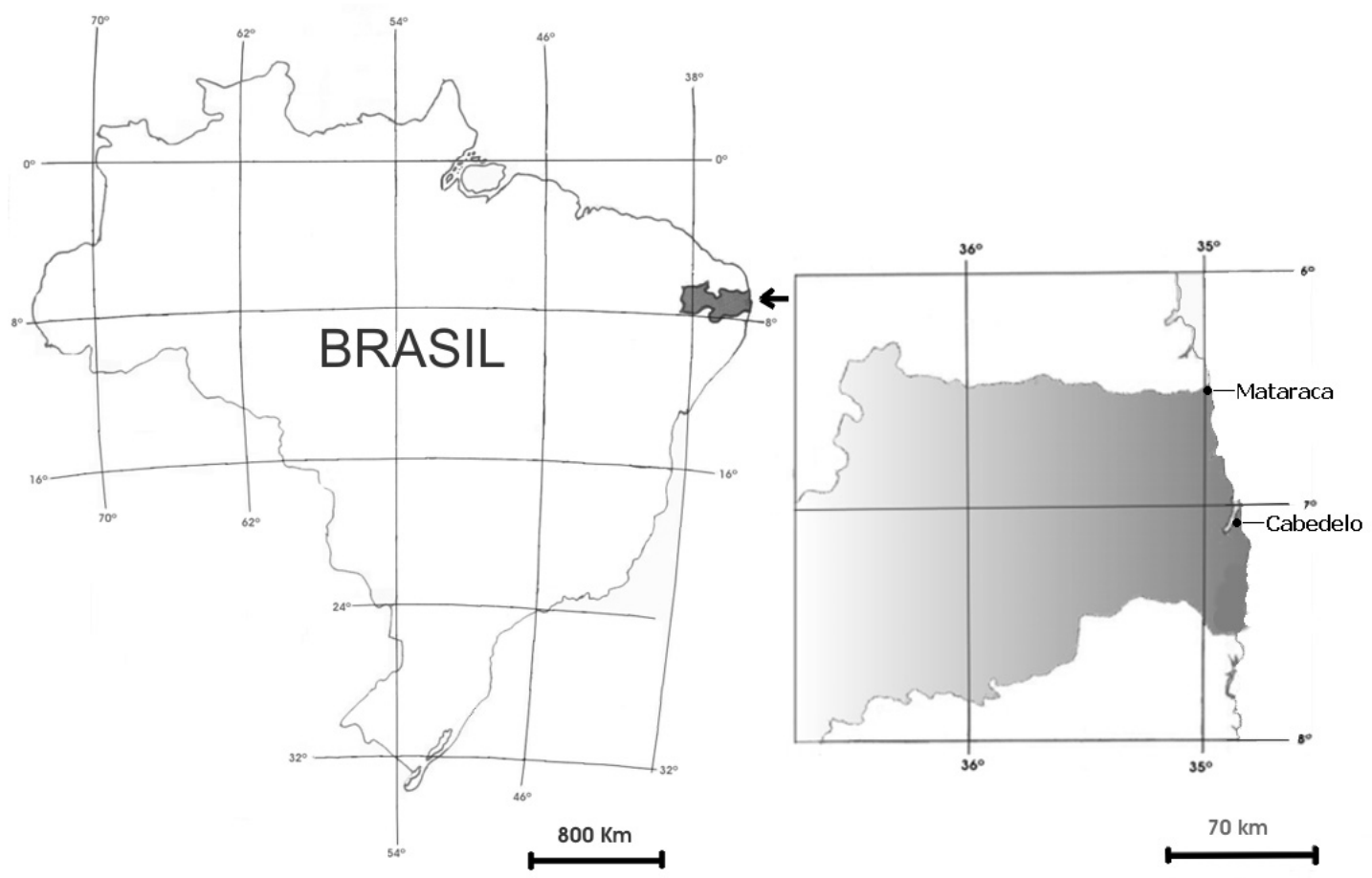

Fig. 1. Localização das duas florestas de restinga no litoral do Estado da Paraíba, Brasil. Os pontos negros, de baixo para cima, representam a Mata da AMEM (Cabedelo) e a área da Mineradora Millennium Inorganic Chemicals do Brasil S.A. (Mataraca). 
Para estimar a riqueza potencial de espécies em cada área foi utilizado o teste Jackknife (HELTSHE \& ForRESTER, 1983). Esse teste foi considerado por PALMER (1990) como o mais apurado estimador não-paramétrico de riqueza de espécies. Também foi construída para cada área uma curva média de acumulação de espécies após simulação aleatória de 15 curvas.

\section{RESULTADOS E DISCUSSÃO}

Vinte e cinco espécies de cupins foram encontradas nas florestas de restinga estudadas, sendo $15 \mathrm{em}$ Cabedelo e 19 em Mataraca, com nove espécies ocorrendo simultaneamente em ambas as áreas. Nas duas localidades, a família Termitidae foi dominante (20 espécies, $80 \%$ ), seguida por Kalotermitidae (3 espécies, $12 \%$ ), e Rhinotermitidae (2 espécies, $8 \%$ ). Entre as subfamílias, Nasutitermitinae foi dominante em número de espécies e freqüência de ocorrência nas parcelas (tab. I). Duas espécies, Nasutitermes coxipoensis (Holmgren, 1910) e Constrictotermes cyphergaster (Silvestri, 1901), foram coletadas ocasionalmente fora dos transectos, em áreas de restinga arbustiva, do tipo "moita", adjacentes às áreas de estudo. Essas espécies aparentemente ocorrem apenas em áreas de formação savânica, sendo a primeira típica do cerrado e a segunda, mais amplamente distribuída, ocorre tanto no cerrado como na caatinga
(Domingos et al., 1986; SENA et al., 2003).

A riqueza potencial de espécies estimada para Cabedelo e Mataraca foi de $J=18,8 \pm 1,8$ e $J=27,7 \pm 3,2$, respectivamente, não havendo diferença significativa nas estimativas ao nível de $95 \%$. A tendência para uma maior riqueza de espécies em Mataraca também pode ser observada na curva acumulativa (fig. 2). A menor riqueza de espécies em Cabedelo pode estar relacionada ao tamanho e isolamento da área, tendo em vista sua localização no perímetro urbano. Em geral, as comunidades termíticas são drasticamente simplificadas, com extinção local de várias espécies, quando seus habitats são fragmentados ou isolados, seja por causas naturais ou antrópicas (DE SouzA \& BRown, 1994; SENA et al., 2003).

As espécies construtoras de ninhos conspícuos nas restingas são: Armitermes holmgreni Snyder, 1926, Microcerotermes exiguus (Hagen, 1858), M. strunckii (Sörensen, 1884), Nasutitermes corniger (Motschulsky, 1855), N. ephratae (Holmgren, 1910) e N. macrocephalus (Silvestri, 1903). A abundância de ninhos (todos arborícolas) foi bem diferente nas duas áreas. Em Cabedelo, as construções eram bem mais comuns, com 19 a 45 ninhos/ha, enquanto em Mataraca os ninhos eram menos abundantes, com 10 a 25 ninhos/ha. Destacaramse as espécies $M$. exiguus e $N$. corniger, pela abundância de suas construções nas duas áreas. Em Mata Atlântica

Tabela I. Riqueza de espécies e número de ocorrências de cupins em duas florestas de restinga do nordeste brasileiro (Br, Brejo de Altitude; Ca, Caatinga; Ce, Cerrado; FA, Floresta Amazônica; MA, Mata Atlântica).

\begin{tabular}{|c|c|c|c|c|c|}
\hline \multirow{2}{*}{ Espécies } & \multirow{2}{*}{ Mataraca } & \multirow{2}{*}{ Cabedelo } & \multicolumn{2}{|c|}{ hábito } & \multirow{2}{*}{ distribuição } \\
\hline & & & nidificação & alimentar & \\
\hline \multicolumn{6}{|l|}{ KALOTERMITIDAE } \\
\hline Neotermes sp. & 0 & 1 & Madeira & Madeira & $?$ \\
\hline Rugitermes sp. & 2 & 0 & Madeira & Madeira & MA \\
\hline Tauritermes sp. & 2 & 0 & Madeira & Madeira & $?$ \\
\hline \multicolumn{6}{|l|}{ RHINOTERMITIDAE } \\
\hline Coptotermes testaceus (Linnaeus, 1758) & 0 & 4 & Subterrâneo & Madeira & $\mathrm{FA} ; \mathrm{MA} ; \mathrm{Ca}$; Ce \\
\hline Heterotermes longiceps (Snyder, 1924) & 11 & 10 & Subterrâneo & Madeira & $\mathrm{MA} ; \mathrm{Br} ; \mathrm{Ca} ; \mathrm{Ce}$ \\
\hline \multicolumn{6}{|l|}{ TERMITIDAE } \\
\hline \multicolumn{6}{|l|}{ Apicotermitinae } \\
\hline Anoplotermes sp. A & 0 & 10 & Subterrâneo & Húmus & MA \\
\hline Anoplotermes sp. B & 3 & 0 & Subterrâneo & Húmus & $?$ \\
\hline \multicolumn{6}{|l|}{ Nasutitermitinae } \\
\hline Armitermes holmgreni Snyder, 1926 & 2 & 1 & Arborícola & Madeira/Húmus & FA; MA; Br \\
\hline Atlantitermes stercophilus Constantino \& De Souza, 1997 & 1 & 0 & $?$ & Madeira & $\mathrm{Br} ; \mathrm{Ce}$ \\
\hline Diversitermes cf. castaniceps (Holmgren, 1910) & 1 & 5 & Serapilheira & Madeira/Folha & $\mathrm{MA} ; \mathrm{Br}$ \\
\hline Nasutitermes cf. minor (Homgren, 1906) & 7 & 0 & Serapilheira & Madeira/Folha & FA \\
\hline Nasutitermes corniger (Motschulsky, 1855) & 4 & 17 & Arborícola & Madeira & $\mathrm{FA} ; \mathrm{MA} ; \mathrm{Br} ; \mathrm{Ca}$ \\
\hline Nasutitermes ephratae (Holmgren, 1910) & 0 & 4 & Arborícola & Madeira & FA;MA; Br \\
\hline Nasutitermes gaigei Emerson, 1925 & 0 & 7 & Madeira & Madeira & FA; MA; Ce \\
\hline Nasutitermes kemneri Snyder \& Emerson, 1949 & 1 & 0 & Madeira & Madeira & $\mathrm{Ca} ; \mathrm{Ce}$ \\
\hline Nasutitermes macrocephalus (Silvestri, 1903) & 1 & 0 & Arborícola & Madeira & $\mathrm{FA} ; \mathrm{MA} ; \mathrm{Ca} ; \mathrm{Ce}$ \\
\hline Nasutitermes sp. & 1 & 0 & Serapilheira & Madeira & $?$ \\
\hline Subulitermes sp. & 2 & 1 & Inquilino & Húmus & $\mathrm{FA} ; \mathrm{Ce}$ \\
\hline Velocitermes sp. & 0 & 3 & Serapilheira & Madeira/Folha & $?$ \\
\hline \multicolumn{6}{|l|}{ Termitinae } \\
\hline Amitermes amifer Silvestri, 1901 & 9 & 16 & Madeira? & Madeira/Húmus & $\mathrm{MA} ; \mathrm{Ca}$; Ce \\
\hline Cylindrotermes cf. nordenskioldi Holmgren, 1906 & 3 & 3 & Madeira & Madeira & $\mathrm{Ce}$ \\
\hline Microcerotermes exiguus (Hagen, 1858) & 19 & 9 & Arborícola & Madeira & FA; MA; Ce \\
\hline Microcerotermes strunckii (Sörensen, 1884) & 1 & 0 & Arborícola & Madeira & $\mathrm{FA} ; \mathrm{Ce}$ \\
\hline Microcerotermes sp. & 2 & 1 & $?$ & Madeira & MA \\
\hline Termes medioculatus Emerson, 1949 & 1 & 0 & Inquilino & Madeira/Húmus & $\mathrm{Fa} ; \mathrm{MA}$ \\
\hline Total de espécies & 19 & 15 & & & \\
\hline Total de ocorrências & 73 & 92 & & & \\
\hline
\end{tabular}




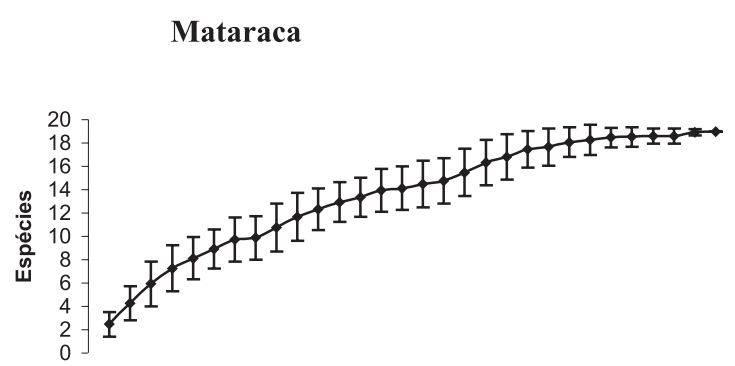

Cabedelo

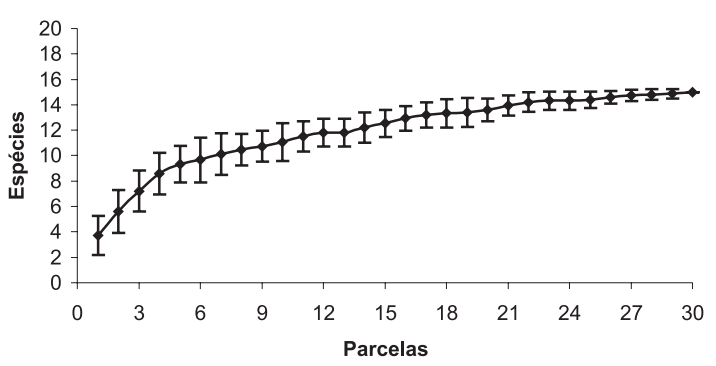

Fig. 2. Curva média ( \pm desvio padrão) de acumulação de espécies em duas florestas de restinga do nordeste brasileiro.

do nordeste brasileiro ao norte do rio São Francisco, o número de ninhos conspícuos de cupins pode ser superior a 80/ha, com grande dominância dos ninhos arborícolas de $M$. exiguus e $N$. corniger (A.Vasconcellos, dados não publicados).

Os cupins foram encontrados principalmente associados à madeira morta da serapilheira, sendo os consumidores de madeira dominantes em riqueza de espécies e em frequiência de ocorrência nas parcelas. O número reduzido de espécies e a baixa freqüência de encontros de cupins da subfamília Apicotermitinae e do grupo alimentar dos humívoros aparentemente estão relacionados com as propriedades do solo. Os solos das restingas são arenosos e pobres em argila e em matéria orgânica, além de possuírem baixa capacidade de retenção de água e nutrientes (ARAUJO \& LACERDA, 1987). Em geral, solos arenosos proporcionam pouca estabilidade para construção de túneis e ninhos subterrâneos pelos cupins, que aparentemente preferem solos mais argilosos (LEE \& Wood, 1971).

A flora e a fauna de vertebrados de restingas no Brasil são constituídas principalmente por componentes de outras formações vegetais (RIzZINI, 1997; ARAUJo \& Lacerda, 1987; Rocha, 2000; Cerqueira, 2000). Seguindo esse mesmo padrão, as espécies de cupins encontradas nas duas florestas de restinga estudadas ocorrem em outros ecossistemas, sendo algumas típicas de formações savânicas, como Nasutitermes kemneri (Snyder \& Emerson, 1949), e de florestas pluviais, como Armitermes holmgreni e $N$. ephratae. A maioria das outras espécies registradas possui ampla distribuição geográfica, sendo sua composição influenciada principalmente pelas espécies de Mata Atlântica, Floresta Amazônia e Cerrado. Mesmo havendo uma forte influência de outras formações vegetais sobre a sua fauna, há duas espécies consideradas endêmicas de restinga, Araujotermes caissara Fontes, 1982 e Atlantitermes guarinim Fontes, 1979 (FonTES 1979; 1982).

Apesar da grande distância entre a Floresta Amazônica e as restingas do nordeste brasileiro, a semelhança entre suas faunas deve-se provavelmente à conexão pretérita entre a Floresta Amazônica e a Mata Atlântica, possivelmente antes do Pleistoceno (BigAReLLA et al., 1975; ANDRADE-Lima, 1982; VIVO, 1997).

Agradecimentos. À Mineradora Millennium Inorganic Chemicals do Brasil S.A., pelo apoio logístico e bolsa de iniciação científica a Eusébio de Moura Vasconcelos Segundo; a Maria Cristina Madeira da Silva, pela leitura crítica e sugestões ao texto; a Akemi Shibuya, pela confecção do mapa; ao IBAMA/CEMAVE$\mathrm{PB}$, pela autorização de coleta na Mata da AMEM; ao CNPq, pela bolsa DCR a Alexandre Vasconcellos (Proc. 309847/20036); e à UEFS, pela bolsa de doutorado a Ana Cerilza S. Mélo.

\section{REFERÊNCIAS BIBLIOGRÁFICAS}

Araujo, D. S. D. \& Lacerda, L. D. 1987. A natureza das restingas. Ciência Hoje 6(33):42-48.

Andrade-Lima, D. 1982. Present-day forest refuges in Northeastern Brazil. In: Prance, G. T. ed. Biological diversification in the tropics. New York, Columbia University Press. p.245-251.

BANDEIRA, A. G. 1989. Análise da termitofauna (Insecta, Isoptera) de uma floresta e de uma pastagem na Amazônia Oriental, Brasil. Boletim do Museu Paraense Emílio Goeldi, Série Zoologia, 5:225-241.

Bandeira, A. G. \& Vasconcellos, A. 2002. A quantitative survey of termites in a gradient of disturbed highland forest in Northeastern Brazil. Sociobiology 39(3):429-439.

Bandeira A. G.; Vasconcellos, A.; Silva, M. P. \& Constantino, R. 2003. Effects of habitat disturbance on the termite fauna in a highland humid forest in the Caatinga domain, Brazil. Sociobiology 42(1):117-127.

Bigarella, J. J. 1965. The Barrreiras group in Northeastern Brazil. Anais da Academia Brasileira de Ciências 47 (Supl.):365-392.

Bigarella, J. J.; AndRade-Lima, D. \& Riehs, P. J. 1975. Considerações a respeito das mudanças paleoambientais na distribuição de algumas espécies vegetais e animais no Brasil. Anais da Academia Brasileira de Ciências 47(Supl.):411-464.

Bignell, D. E. \& Eggleton, P. 2000. Termites in ecosystems. In: Abe, T.; Higashi, M. \& Bignell, D. E. eds. Termites: Evolution, sociality, symbiosis, ecology. Dordrecht, Kluwer Academic Publications. p.363-387.

Carvalho, M. G. R. F. 1982. Estado da Paraíba - Classificação geomorfológica. João Pessoa, Editora Universitária-UFPB. 77p.

Cerqueira, R. 2000. Biogeografia das restingas. In: Esteves, F. A. \& Lacerda, L. D. eds. Ecologia de Restingas e Lagoas Costeiras. Macaé, NUPEM/UFRJ. p.65-75.

Constantino, R. 1992. Abundance and diversity of termites (Insecta: Isoptera) in two sites of primary rain forest in Brazilian Amazonia. Biotropica 24(3):420-430.

1998. Catalog of the living termites of the new world (Insecta: Isoptera). Arquivos de Zoologia 35:135-231.

1999. Chave ilustrada para identificação dos gêneros de cupins (Insecta, Isoptera) que ocorrem no Brasil. Papéis Avulsos de Zoologia 40(25):387-448.

De Souza, O. F. F. \& Brown, V. B. 1994. Effects of habitat fragmentation on Amazonian termites communities. Journal of Tropical Ecology 10:197-206.

Domingos, D. J.; Cavenaghi, T. M. C. M.; Gontio, T. A.; Drumond, M. A. \& Carvalho, R. C. F. 1986. Composição em espécies, densidade e aspectos biológicos da fauna de cupins de cerrado em Seta Lagoas-MG. Ciência e Cultura 38(1):1-9.

FonTES, L. R. 1979. Atlantitermes, novo gênero de cupim, com duas novas espécies do Brasil (Isoptera, Termitidae, Nasutitermitinae). Revista Brasileira de Entomologia 23(4):219-227.

1982. Novos táxons e novas combinações nos cupins 
nasutos geófagos da região neotropical (Isoptera, Termitidae, Nasutitermitinae). Revista Brasileira de Entomologia 26(1):99-108.

Governo do Estado da Paraíba, 1985. Atlas Geográfico do Estado da Paraíba. João Pessoa, Grafset. 100p.

Heltshe, J. F. \& Forrester, N. E. 1983. Estimating species richness using the jackknife procedure. Biometrics 39:1-11.

Lavelle, P.; Bignell, D. \& Lapage, M. 1997. Soil function in changing world: the role of invertebrate ecosystems engineers. European Journal Soil Biology 33(4):159-193.

Lee, K. E. \&. Wood, T. G. 1971. Termites and soils. London, Academic. 251p.

Mabesoone, J. M. \& Castro, C. 1975. Desenvolvimento geomorfológico do Nordeste Brasileiro. Boletim do Núcleo Nordeste da Sociedade Brasileira de Geologia 3:5-35.

Матsumoto, Т. 1976. The role of termites in an equatorial rain forest ecosystem of west Malaysia: population density, biomass, carbon, nitrogen and calorific content and respiration rate. Oecologia 22:153-178.

NIMER, E. 1979. Climatologia do Brasil. Rio de Janeiro, IBGE. 421p. Oliveira-Filho, A. T. \& Carvalho, D. A. 1993. Florística da vegetação do extremo norte litoral da Paraíba. Revista Brasileira de Botânica 16(1):115-130.

Palmer, M. W. 1990. The estimation of species richness by extrapolation. Ecology 71:1195-1199.

Rizzinı, C. T. 1997. Tratado de fitogeografia do Brasil. Rio de Janeiro, Âmbito Cultural. 747p.

Rocha, C. F. D. 2000. Biogeografia de répteis de restingas: distribuição, ocorrência e endemismo. In: Esteves, F. A. \& Lacerda, L. D. eds. Ecologia de Restingas e Lagoas Costeiras. Macaé, NUPEM/UFRJ. p.99-116.

Sena, J. M.; Vasconcellos, A.; Gusmão, M. A. B. \& Bandeira, A. G. 2003. Assemblage of termites in a fragment of cerrado on the coast of Paraiba State, Northeast Brazil (Isoptera). Sociobiology 42(3):753-760.

Vivo, M. 1997. Mammalian evidence of historial ecological change in the caatinga semiarid vegetation of northeastern Brazil. Jounal of Comparative Biology 2:65-73.

Wood, T. G. \& SAnds, W. A. 1978. The role of termites in ecosystems. In: Brian, M. V. ed. Production ecology of ants and termites. Cambridge, Cambridge University Press. p.245-292.

Recebido em junho de 2004. Aceito em abril de 2005. ISSN 0073-4721 Artigo disponível em: www.scielo.br/isz 